\title{
The effect of aging on the frequency, phenotype and cytokine production of human blood CD4 + CXCR5 + T follicular helper cells: comparison of aged and young subjects
}

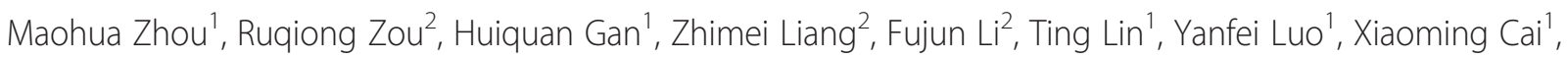
Fang $\mathrm{He}^{3}$ and Erxia Shen ${ }^{2^{*}}$

\begin{abstract}
Background: T cell-dependent B-cell responses decline with age, indicating declined cognate helper activity of aged CD4 + T cells for B cells. However, the mechanisms remain unclear. T follicular helper (Tfh) cells, a novel T helper subset, play an essential role in helping B cells differentiation into long-lived plasma cells in germinal center (GC) or short-lived plasma cells. In the present study, we proposed that there might existe changes of proportion, phenotype or cytokine production of blood Tfh cells in healthy elderly individuals compared with healthy young individuals.
\end{abstract}

Results: The results showed that frequencies of aged blood CXCR5 + CD4 + Tfh cells increased compared with young subjects. Both aged and young blood CXCR5 + CD4 + Tfh cells constitutively expressed CD45RO, CCR7 and CD28, and few of these cells expressed CD69 or HLA-DR, which indicated that they were resting memory cells. There was no significant difference of IL-21 frequency production by aged blood CXCR5 + CD $4+$ Tfh determined by FACS compared with young individuals, however, aged PBMCs produced significantly higher levels of IL-21 evaluated by ELISA. Furthermore, there were no significant differences of percentages of IFN- $\gamma$, IL-4, IL-17 or IL-22 production by aged Tfh cells compared with their counterparts of young individuals respectively. However, frequencies of IL-17+ cells within aged CD4 + CXCR5-T cells were markedly lower than in the young individuals. Furthermore we observed different frequencies of IFN- $\gamma$, IL-17, IL-4 or IL-22 production by Tfh or by CD4 + CXCR5- cells in aged and young subjects respectively.

Conclusions: Our data demonstrated that the frequencies of blood memory CXCR5 + CD4 + Tfh cells increased in the

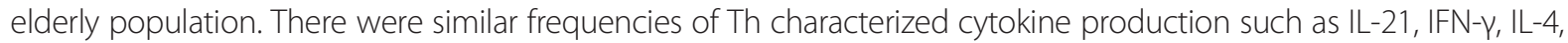
IL-17 or IL-22 in aged and young Tfh cells. However, aged PBMCs produced a significantly higher amount of IL-21 compare to young subjects.

Keywords: Tfh cells, Aging, IL-21

\footnotetext{
* Correspondence: erxia_shen@yahoo.com

${ }^{2}$ Department of Pathogenic Biology and Immunology, Guangzhou Hoffmann Institute of Immunology, School of Basic Sciences, Guangzhou Medical University, Guangzhou 510182, China

Full list of author information is available at the end of the article
} 


\section{Introduction}

The immune system undergoes significant changes with aging, which likely partly contributes to increased susceptibility to infections, malignancies, inflammatory diseases, and reduced response to vaccination of elderly individuals [1,2]. Significant decline in immune function during aging is termed immunosenescence [3], and higher frequencies of inflammatory diseases of elderly people indicate that aging could be regarded as a condition of dys-regulated inflammation [4].

Age-associated alterations in immune dysfunction are found in both innate and adaptive immunity in humans. For example, age-associated changes in NK cell phenotype have been previously reported that can be responsible of functional NK cell deficiency [5,6]. DCs from elderly individuals are compromised in response to microorganisms but display increased reactivity to self-antigens associated with chronic inflammation and autoimmunity [7-9]. Most importantly, profound changes in $\mathrm{T}$ cell function and subsets are observed in elder individuals $[10,11]$.

Elderly population suffers increased morbidity and mortality from infectious diseases, therefore, the elderly are highly encouraged to receive vaccinations for these infections such as influenza and pneumococcal pneumonia. However, the efficacy of vaccination reduces with aging [12-14]. The mechanisms of increased infections and declined response to vaccination partially due to declined humoral response which are mainly due to function defects in cognate helper function of $\mathrm{CD} 4+\mathrm{T}$ cells and $\mathrm{B}$ cells. Accumulating evidence demonstrated that the genesis, numbers and function of $\mathrm{B}$ cell altered with aging- [15-19]. Declined cognate helper activity of aged $\mathrm{CD} 4+\mathrm{T}$ cells for B cells in mice were observed [20-22]. However, this phenomenon and mechanisms are not well understoodin human beings.

A distinct subset of CD4+ helper $\mathrm{T}$ (Th) cells, Tfh cell (Tfh) specialize in providing cognate help to antigenspecific B cells in germinal centers (GCs). Tfh cells play a key role in the formation of GCs and differentiation of GC B cells into plasma cells and memory B cells [23,24]. In GCs, Tfh cells express master regulator transcription factor $\mathrm{Bcl} 6$ for their generation and surface marker CXCR5 which facilitates Tfh cells homing to B cell follicles $[25,26]$. IL-21 is an important effector cytokine secreted by Tfh cells and directly regulates B cell proliferation and class switching [27]. However, it is extremely challenging to obtain lymph node samples from humans to define the role of Tfh cells in normal or diseases conditions. Therefore, one study published on immunity evaluated the relationship of blood CXCR5 + CD $4+T$ cells with Tfh cells, and demonstrated that human blood CXCR5 + CD4 + T cells shared functional properties with Tfh cells, and appeared to represent their circulating memory compartment [28].
Therefore, in this study we determined whether there were alterations in aged Th cells by detecting blood CXCR5 + CD $4+\mathrm{T}$ cells which represented Tfh cells in blood. And we observed CD69 and HLA-DR expression on CXCR5 + CD $4+$ Tfh cells from young or aged individuals respectively. Furthermore, Th characterized cytokine IL-21, IFN- $\gamma$, IL-4, IL-17 or IL-22 secreted by age or young Tfh cells were determined and compared.

\section{Results \\ Increased proportion of peripheral blood CXCR5 + CD4 + Tfh cells from the elderly individuals}

First, we wanted to demonstrate whether cell percentages or numbers of CXCR5 + CD 4 + Tfh cells changed in the elderly or not. The results showed that there was no significant difference of lymphocyte counts (Figure 1A), frequencies or cell counts of CD4 $+\mathrm{T}$ cells (Figure 1B, $1 \mathrm{C})$ between young and aged healthy individuals. Interestingly, frequencies of CXCR5 + CD4 + Tfh cells (18.90 \pm $1.004 \%)$ from elderly individuals were significantly higher compared with those from young people (15.71 $\pm 0.608 \%)$, and correlated with age. Furthermore, the percentages of blood Tfh cells from young children were the lower than elder individuals (Figure 1D and $1 F$ ). In addition, there was no significant difference of cell counts of CXCR5 + CD4 + Tfh cells between aged and young subjects (Figure 1E). These results demonstrated that aged blood CXCR5 + CD4 + Tfh cells proportion increased compared with young subjects.

\section{Memory phenotype CD45RO and CCR7, and co-stimulatory CD28 expression of aged blood CXCR5 + CD4 + Tfh cells}

We evaluated whether there were some kind of differences in the memory phenotype of CXCR $5+\mathrm{CD} 4+$ Tfh cells from aged individuals compared with young individuals. First, we determined the memory phenotype CD45RO and CCR7 expression on CXCR5 + CD4 + Tfh cells using FACS. Both aged and young CXCR5 + CD $4+$ Tfh cells constitutively expressed CD45RO and CCR7 (Figure 2A and $2 \mathrm{~B}$ ), and there was no significantly difference between healthy aged and young individuals (Figure $3 \mathrm{~A}$ and $3 \mathrm{~B}$ ). In addition, higher percentages of CD4 + CD45RO+, CD3 + CD4-CD45RO+, and CD4 + CXCR5-CD45RO + memory $\mathrm{T}$ cells were observed in aged individuals (Figure $3 \mathrm{~A}$ ). In contrast, frequencies of CCR7 positive cells among CD4+, CD3 + CD4- and CD4 + CXCR5-T cells from aged individuals decreased, and there was significant difference of percentages of CCR7 + CD3 + CD4-Tcells of elderly subjects compared with young subjects (Figure $3 \mathrm{~B}$ ).

Meanwhile, we determined co-stimulatory molecule CD28 expression on blood CXCR5 + CD $4+$ Tfh cells using FACS. Percentages of CD28 positive cells among CD4+, CD3 + CD4- and CD4 + CXCR5-T cells from elderly individuals significantly decreased compared with 

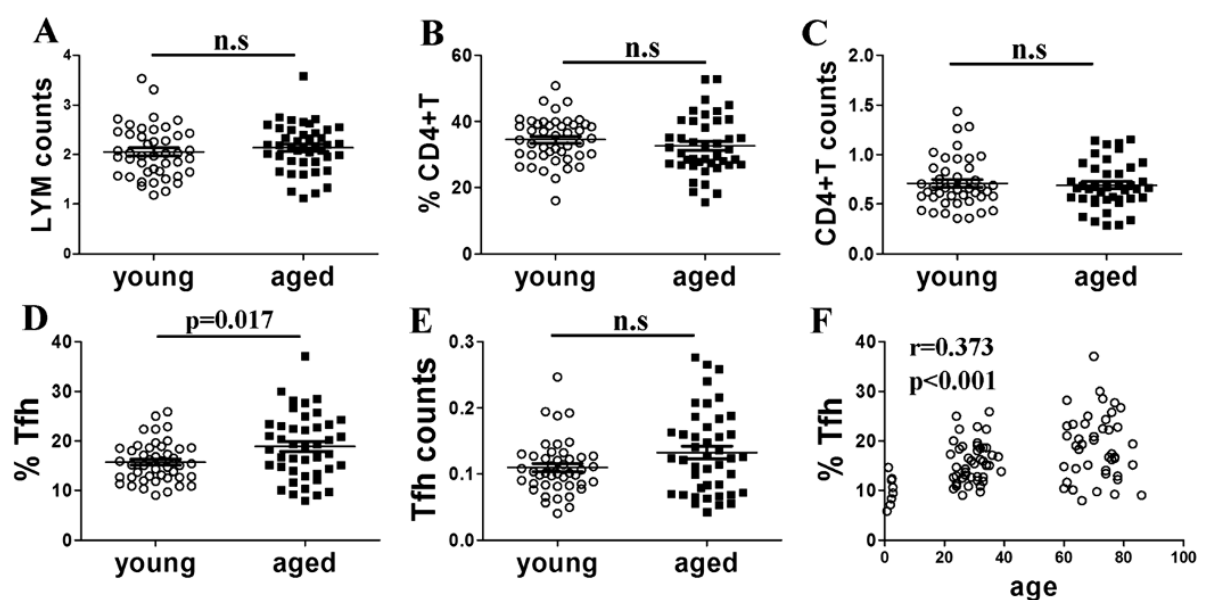

Figure 1 Increased proportion of blood CXCR5 + CD4 + Tfh cells of aged subjects. A. LYM counts: lymphocyte counts of peripheral blood $\left(10^{9} / \mathrm{L}\right)$. B. Percentages of CD4 + T cells $(C D 3+C D 4+)$ in lymphocytes from young and aged groups determined by FACS. C. Cell numbers of CD4 $+\mathrm{T}$ cells in peripheral blood $\left(10^{9} / \mathrm{L}\right)$. D. Frequencies of Tfh cells (CXCR5 + CD4+) in CD4 + T cells determined by FACS. E. The total cell counts of Tfh cells $(C X C R 5+C D 4+)$ in peripheral blood $\left(10^{9} / \mathrm{L}\right)$. F. Correlation of percentages of CXCR5 + CD $4+$ Tfh cells with age. One open circle or filled square represented one individual. Data were expressed with line at mean with SEM.

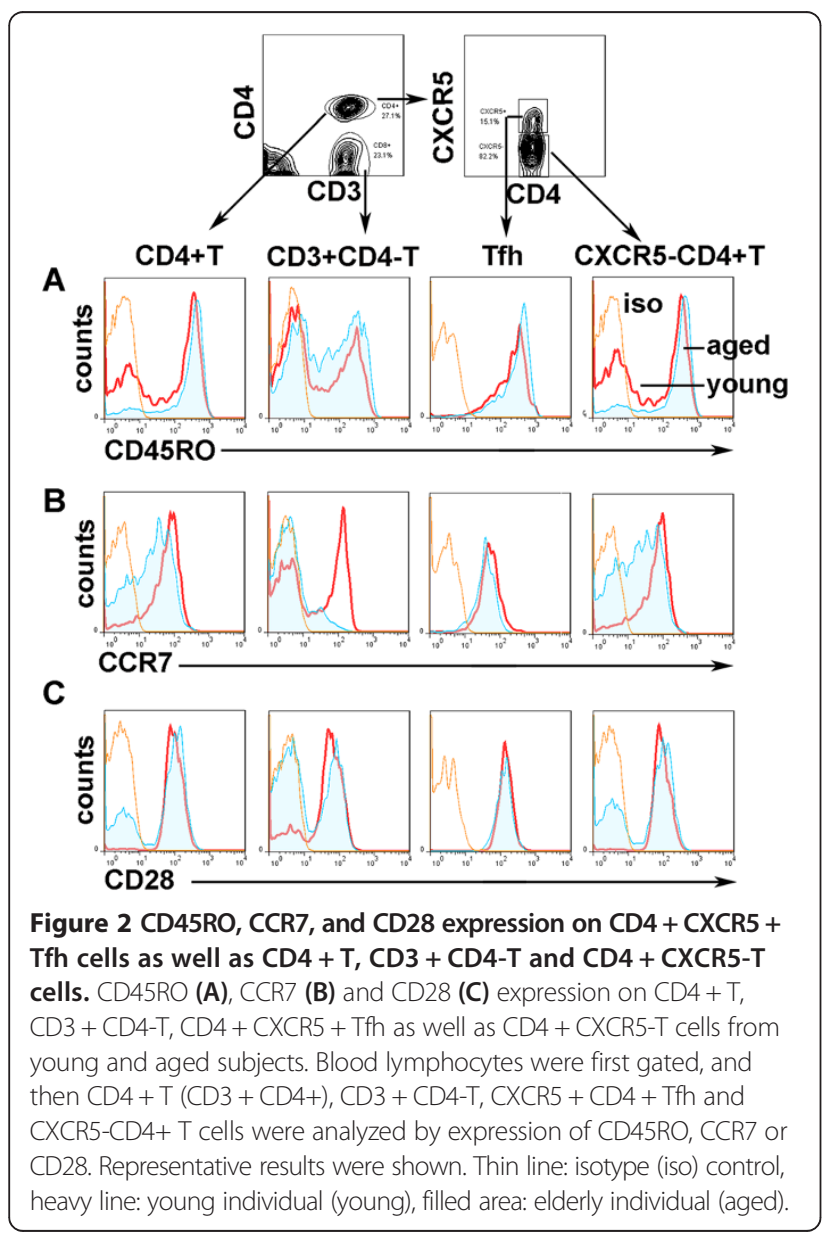

their counterparts of young individuals (Figures 2C and $3 \mathrm{C}$ ). However, there were no differences of frequencies of $\mathrm{CD} 28$ expression between aged and young CXCR5 + CD4 + Tfh cells. Taken together, these results indicated that Tfh cells constitutively expressed CD45RO and CCR7, and were peripheral memory T cells.

Both aged and young blood Tfh cells are in resting state Next, early activation marker CD69 and late activation marker HLA-DR expression on Tfh and other T subsets from young and aged individuals were evaluated by FACS. The results showed frequencies of CD69+ cells among CXCR5 + CD4 + Tfh cells, as well as CD4+T, CD3 + CD4-T and CD4 + CXCR5-T cells from healthy elderly individuals had no significant change compared with their counterparts of healthy young individuals (Figure 4A). In addition, there were different proportions of CD69+ cells among CD4+ and CD3 + CD4-T cells both from healthy young and aged subjects. Similar with CD69 expression, few of CD4+ or CD3 + CD4-T cells subsets expressed HLA-DR, and there were no significant differences of HLA-DR expression on CXCR5 + CD4 + Tfh cells as well as on CD4 + T, CD3 + CD4-T and CD4 + CXCR5-T cells from young subjects and aged individuals (Figure 4B). Above results demonstrated that both aged and young blood CXCR $5+\mathrm{CD} 4+\mathrm{Tfh}$ cells were in resting state.

Similar frequency of IL-21 produced by aged and young blood CXCR5 + CD4 + Tfh cells

IL-21 is mainly produced by Tfh cells, and it is a very important cytokine of Tfh to help B cells. Therefore, we determined IL-21 production by CXCR $5+\mathrm{CD} 4+\mathrm{Tfh}$ or 

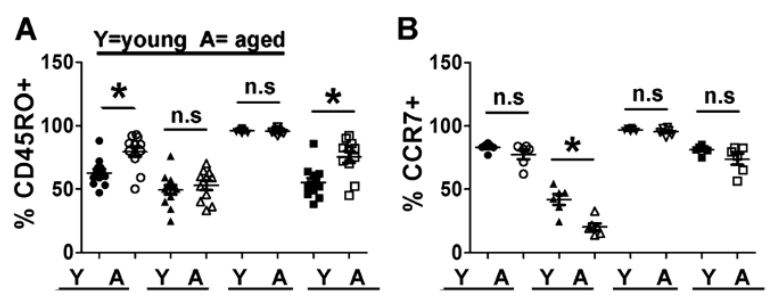

CD4+T CD3+CD4-T Tfh CD4+CXCR5- CD4+T CD3+CD4-T Tfh CD4+CXCR5-

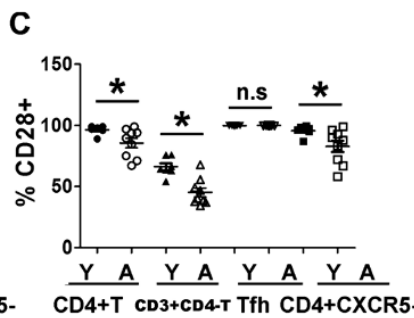

Figure 3 Statistical analysis of CD45RO, CCR7 and CD28 expression on CD4 + CXCR5 + Tfh and other T subsets. Percentages of CD45RO + (A) (young subjects: $n=12$, aged subjects: $n=11$ ), CCR7+ (B) (young subjects: $n=6$, aged subjects: $n=6$ ) and CD28 + (C) (young subjects: $n=7$, aged subjects: $n=9$ ) among CD4 + T, CD3 + CD4-T, CD4 + CXCR5 + Tfh and CD4 + CXCR5-T cells were shown as in histograms. Y: young individuals, A: aged individuals. *: $p<0.05$, n.s: no significant difference. All data were expressed with line at mean with SEM.

CD4 + CXCR5-T cells using intracellular cytokine staining. The results showed that both Tfh and CD4+ CXCR5-T cells could produce IL-21 (Figure 5A), and there was no significant difference of frequencies of IL$21+$ cells inCD $4+$ CXCR5 + Tfh as well as CD4+ CXCR5-T cells from the elderly compared with young individuals (Figure 5B). Meanwhile, PBMCs were prepared from healthy aged and young individuals, and cultured in the absence or presence of PMA and inomycin for 3 days, and then the concentration of IL-21 was determined by ELISA. As shown in Figure 5C, there were very low levels of IL-21 without stimulation from both aged and young individuals. Compared with young subjects, aged PBMCs stimulation with PMA and inomycin produced significantly higher levels of IL-21. These results indicated that IL-21 production by aged PBMCs increased significantly compared with young individuals.

Th1, Th2, Th17 and Th22 characterized cytokine production within CD4 + CXCR5 + Tfh or CD4 + CXCR5-T cells from healthy aged and young individuals Finally, we evaluated Th1, Th2, Th17, and Th22 characterized cytokines IFN- $\gamma$, IL-4, IL-17 or IL-22 production within blood CD4 + CXCR5 + Tfh cells or CD4 + CXCR5-

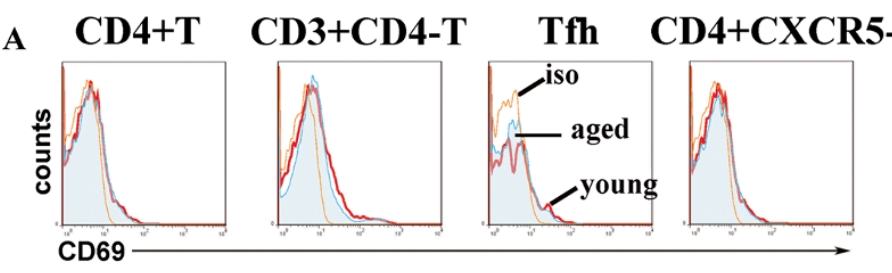

B
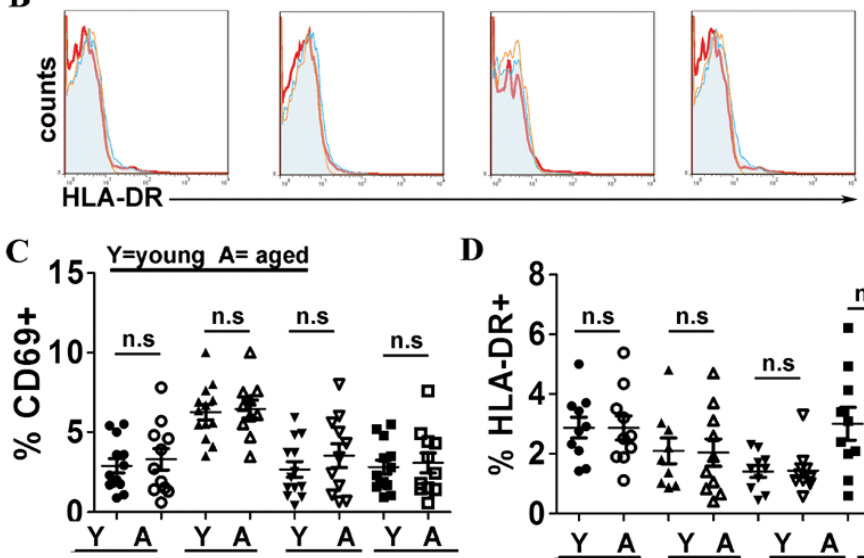

D

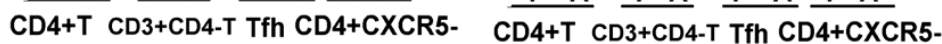

Figure 4 CD69 and HLA-DR expression on young or aged CD4 + CXCR5 + Tfh cells, CD4 + T, CD3 + CD4-T and CD4 + CXCR5-T cells. CD69 (A) and HLA-DR (B) expression on CD4 + T, CD3 + CD4-T, CD4 + CXCR5 + Tfh and CD4 + CXCR5-T cells from representative results were shown as in histograms. Thin line: isotype control (iso), heavy line: young individual (young), filled area: elderly individual (aged). Statistical results of percentages of CD69 + (C) (young subjects: $n=13$, aged subjects: $n=11$ ), and HLA-DR + (D) (young subjects: $n=10$, aged subjects: $n=10)$ among CD4 +T, CD3 + CD4-T, CD4 + CXCR5 + Th and CD4 + CXCR5-T cells were shown. Y: young individuals, A: aged individuals. n.s: no significant difference. All data were expressed with line at mean with SEM. 

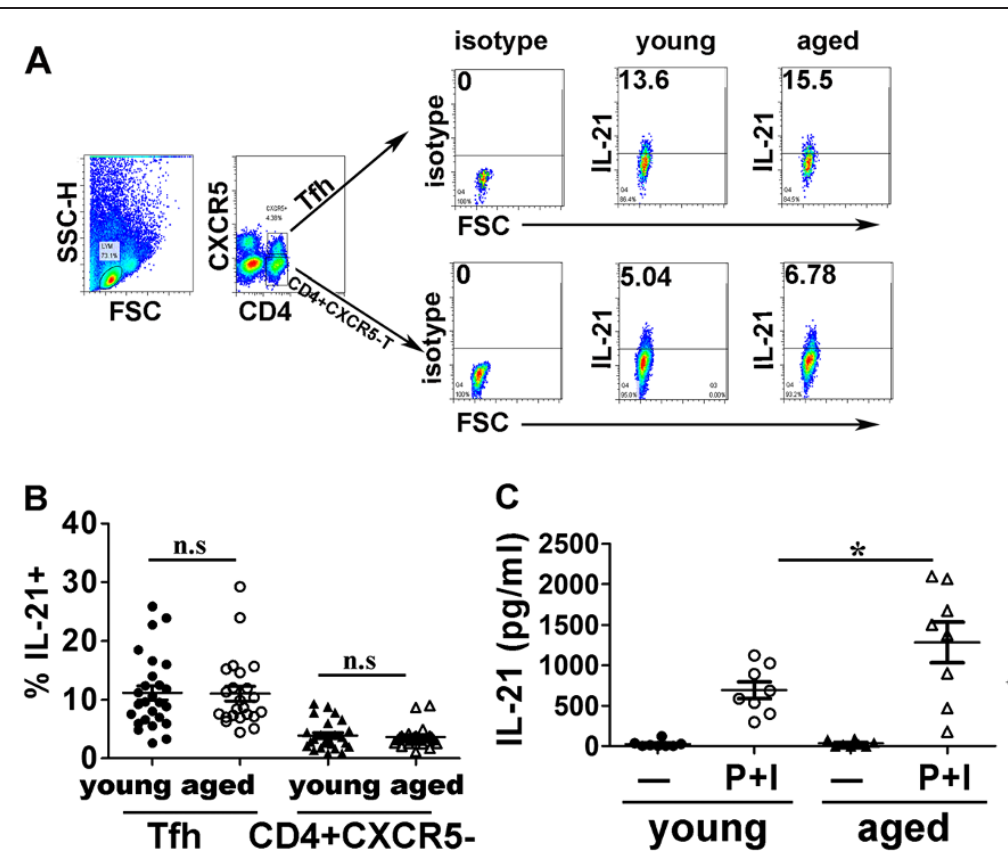

Figure 5 Similar frequency of IL-21 produced by aged and young blood CXCR5 + CD4 + Tfh cells. A. Representative results of IL-21+ cells produced by Tfh or CD4 + CXCR5-cells were shown. B. FACS data of percentages of IL-21+ cells in CD4 + CXCR5 + Tfh cells or CD4 + CXCR5- cells from both aged and young individuals (young subjects: $n=23$, aged subjects: $n=23$ ) were shown. $C$. IL-21 concentration determined by ELISA was shown. PBMCs were prepared and cultured with P + I (PMA and inomycin) or - (medium) for 3 days. Supernatants were collected and IL-21 was evaluated by ELISA. *: $p<0.05$. All data were expressed with line at mean with SEM.
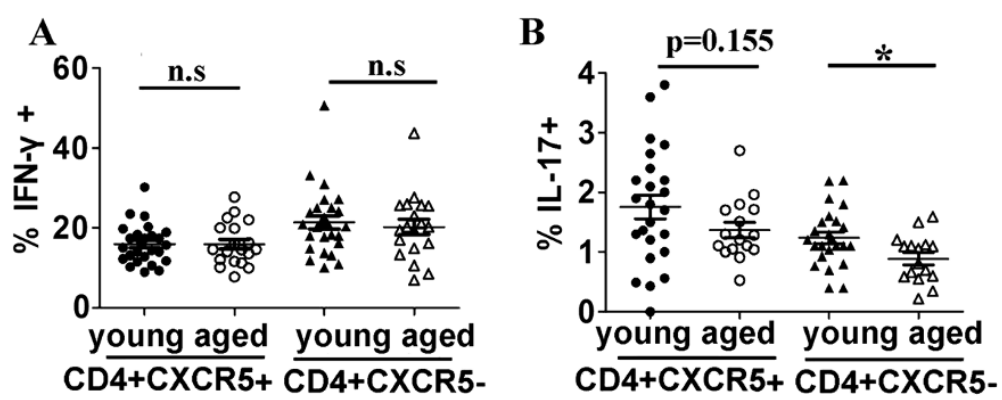

C

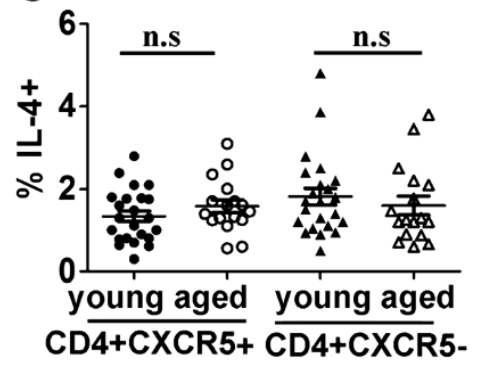

D

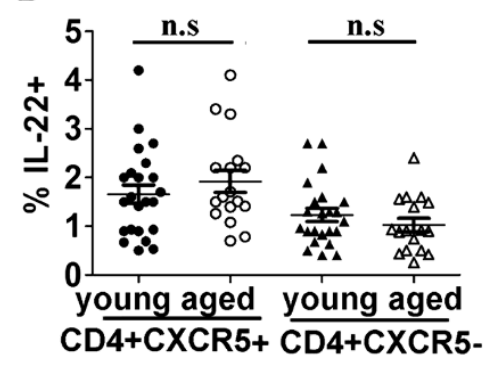

Figure 6 Comparison of IFN- $\gamma+$, IL-17+, IL-4 + or IL-22+ cells among aged and young CXCR5 + CD4 + Tfh. PBMCs were prepared, stimulated with PMA and inomycin for 4-6 hours, and intracellular staining were done. FACS data were analyzed: lymphocytes were first gated and then CD4 + CXCR5 + or CD4 + CXCR5- cells were gated and cytokines such as IFN- $\gamma$, IL-17, IL-4 and IL-22 were analyzed. Statistical data of frequencies of IFN- - (A), IL-17 + (B), IL-4 + (C) and IL-22 + (D) among CD4 + CXCR5 + Tfh or CD4 + CXCR5- cells were shown. *: p $<0.05$, n.s: no significant difference. All data were expressed with line at mean with SEM. 
$\mathrm{T}$ cells from young and elderly subjects. The results showed that there were no significant differences of percentages of IFN- $\gamma+$, IL-4+, or IL- $22+$ cells within aged blood CD4 + CXCR5 + Tfh or CD4 + CXCR5-T cells compared with their counterparts of young individuals respectively (Figure 6A, 6C and 6D). However, frequencies of IL-17 producing cells within aged CD4 + CXCR5-T cells were markedly lower, and there was a slight tendency for IL 17-producing cells to be lower in the elderly $(\mathrm{p}=0.155)$ (Figure 6B). Moreover, lower percentages of IFN- $\gamma+$ or IL-4 production by young blood CXCR $5+$ CD $4+$ Tfh cells, and higher frequencies of IL-17 or IL-22 production by blood young or aged CXCR $5+\mathrm{CD} 4+$ Tfh cells were observed compared with their counterparts within CD4 + CXCR5-T cells, respectively. There was no significant difference of frequencies of IFN- $\gamma$ or IL-4 producing cells within aged CD4 + CXCR5-T cells and aged CXCR5 + CD4+ Tfh cells (Figure 7).

\section{Discussion}

Tfh cells have been recently established as a novel Th subset specialized for providing help to B cells in GCs to differentiate into long-lived memory B and plasma cells [23,24]. Age-related declines in humoral responses are mainly due to defects in the cognate helper function of CD4 + T cells and in the B cells from aged individuals [15-19]. However, this phenomenon and the mechanisms of declined cognate helper activity of aged CD4 $+\mathrm{T}$ cells for $\mathrm{B}$ cells remain not well understood in human beings.
In the present study, we determined whether there are alterations of Tfh cells in aging of human beings.

First, we determined peripheral blood CXCR5 + CD4 + Tfh cells from healthy young and elderly individuals. It is extremely challenging to determine GCs' Tfh cells through obtaining lymph node samples from healthy humans routinely. As demonstrated by one study published on immunity, human blood CXCR5 + CD4 + T cells shared functional properties with Tfh cells and appeared to represent their circulating memory compartment [28]. Therefore, we defined blood CXCR5 + CD4 + T cells as peripheral blood Tfh by FACS. The results demonstrated that frequencies of aged CXCR5 + CD4 + Tfh cells were higher than those of young Tfh cells, and correlated with age (Figure 1). Consistent with our results, aged mice have increased numbers of isolated lymphoid follicles (ILFs), an increased T-lymphocyte population within aged ILFs [29]. We observed that there were no significant differences of percentages or cell counts of CD $4+\mathrm{T}$ cells. Different literatures reported different data about change of CD $4+\mathrm{T}$ number and percentage in the elderly. Hirokawa et al. observed an age-related increase in the number of CD4+ T cells in the Japanese population [30]. Provinciali et al's results showed the absolute number of total CD4+ T cells was progressively reduced with increasing age in Italian population [31]. Lower cell counts not percentages of CD $4+\mathrm{T}$ cells in the elderly in Hongkong were observed [32]. The different data might be due to race,
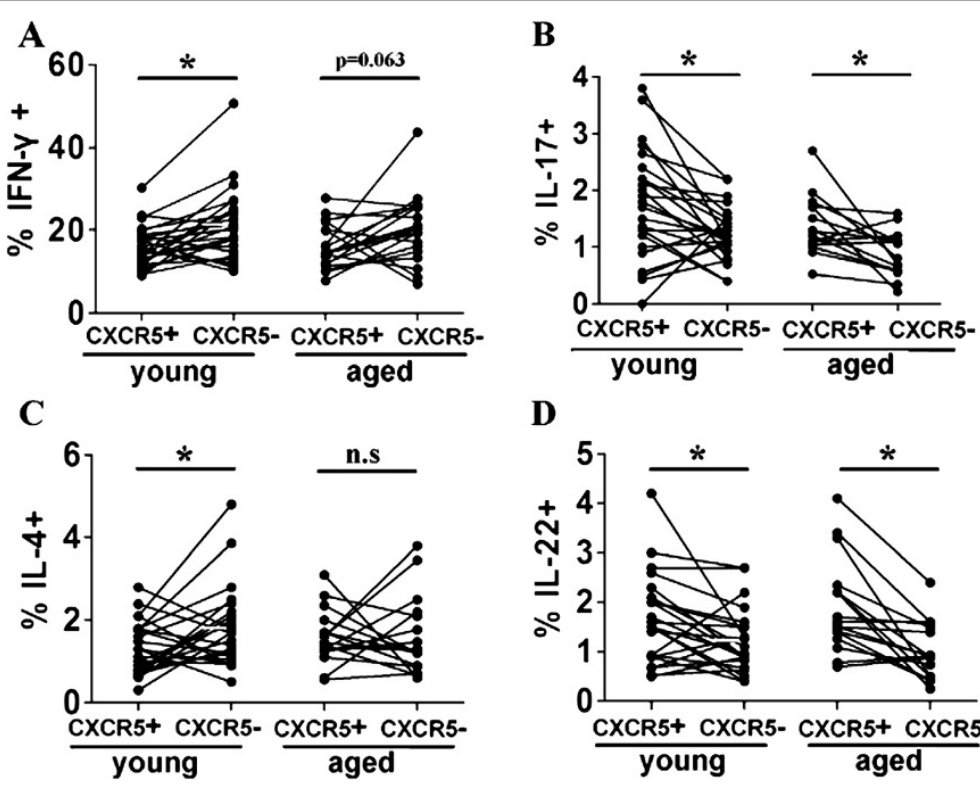

D

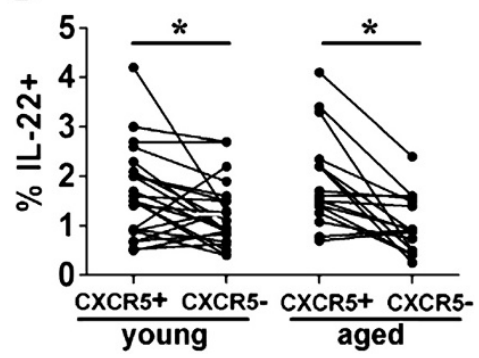

Figure 7 Different frequencies of IFN- -+ , IL-17+, IL-4+ or IL-22+ cells among Tfh compared with CD4 + CXCR5- cells. Statistical data of frequencies of IFN- $\gamma+$ (A), IL-17 + (B), IL-4 + (C) and IL-22 + (D) among CD4 + CXCR5 + Tfh or CD4 + CXCR5- cells were shown. All data were expressed with line at mean with SEM. Differences between two groups were assessed by paired $t$ test for two-tails. *: $p<0.05, n$.s: no significant difference. 
sex, CMV infection, and other factors impact on subpopulation of CD4 + T cells [32,33].

Then, we evaluated and compared the memory phenotype CD45RO and CCR7and co-stimulatory molecule CD28 expression on CXCR5 + CD $4+$ Tfh cells. The results showed that both aged and young blood CXCR5 + CD4 + Tfh cells constitutively expressed CD45RO and CCR7, and there was no difference between young and aged subjects (Figures 2 and 3). As we know, both CD45RO and CCR7 are expressed by central memory T cells [34]. Consistent with previous reports [28,35-37], blood CXCR5 + CD4 + Tfh cells co-expressing CD45RO and CCR7 belong to memory $\mathrm{T}$ cells. In addition, similar with other studies, we observed that aged memory CD4+ $\mathrm{T}$ cells accumulated increased [10,11,38]. Moreover, we demonstrated that blood CXCR5 + CD4 + Tfh cells constitutively expressed co-stimulatory molecule CD28 (Figures 2 and 3). CD28, a major co-stimulatory receptor, was responsible for the optimal antigen-mediated $\mathrm{T}$-cell activation, proliferation and survival of $\mathrm{T}$ cells. Previous reports demonstrated that CD28 was essential for Tfh development [39]. Furthermore, decreased CD28 expression on aged CD4 + T, CD3 + CD4-T or CXCR5-CD4 + T cells was observed in this study. Consistent with our results, the accumulation of CD28 (-) T cells, particularly within the CD8 subset, is one of the most prominent changes during in the age-associated decline of immune function in humans [40]. Blood CXCR5 + CD4 + Tfh cells constitutively expressed CD28, which indicated that these cells could be fully activated and play a key role in development and function of CXCR5 + CD4 + Tfh cells from both aged and young subjects.

IL-21 is the most potent cytokine known for driving B cells differentiation into plasma cells in both mice and humans, and is highly secreted by Tfh cells, although not exclusively secreted by Tfh cells $[27,41,42]$. In this study, we demonstrated aged PBMCs produced significantly higher levels of IL-21compared with young subjects (Figure 5). Consistent with our observation, there has been recently reported that aged $\mathrm{CD} 4+\mathrm{T}$ cells from healthy elderly individuals secreted significantly higher levels of IL-21 on priming with dendritic cells (DC) under anti-CD3 plus anti-CD28 mAbs [43], and IL-21 was mainly produced by memory CD4 $+\mathrm{T}$ cells [44]. There were higher memory CD $4+\mathrm{T}$ cells in aged subjects, therefore there were higher levels of IL-21 produced by aged PBMCs. In contrast, there has been reported that aged memory $\mathrm{CD} 4+\mathrm{T}$ cells expressed lower levels of IL-21 after stimulated with anti-CD3 plus anti-CD28 evaluated by real time RT-PCR [45]. The different results might be due to different experimental condition. IL-21 is a cytokine that has broad effects on immune and non-immune cells, and more and more data suggest this cytokine is critically involved in the initiation and/or progression of inflammatory reactions where self-reactive immune cells or antibodies cause damage in tissue [46]. Increased IL-21 production by aged PBMCs may significantly impact immune functions in elderly individuals, and contribute to aging associated inflammatory condition or diseases.

Recently, there was one literature which reported that human blood CXCR5 + CD4 + Tfh cells comprised three subsets: Th1, Th2 and Th17 cells with different capacity to help B cells. Th2 and Th17 cells within CXCR5+, but not within CXCR5-, compartment efficiently induced naïve $B$ cells to produce Abs. In contrast, Th1 cells from both CXCR5+ and CXCR5- compartments lacked the capacity to help B cells [28]. Our results demonstrated that there were no significant differences of percentages of IFN- $\gamma$, or IL-4 production by aged blood CD $4+$ CXCR $5+$ Tfh or CD4 + CXCR5-T cells compared with their counterparts of young individuals (Figure 6). Previous studies about age-associated change in IFN- $\gamma$ or IL-4 production by $\mathrm{T}$ cells were largely inconsistent [47]. The frequencies of IL-17+ cells produced by aged CD4 + CXCR5-T cells were markedly lower, and frequencies of IL-17 production by aged Tfh cells didn't decrease significantly compared with young subjects (Figure 6B).Consistent with our results, previous study demonstrated that purified memory $\mathrm{CD} 4+\mathrm{T}$ cells from aged people produced lower levels of frequency of Th17 cells compared with the young [48].

\section{Conclusions}

In conclusion, in the present study we observed that frequencies of aged blood CXCR $5+\mathrm{CD} 4+\mathrm{Tfh}$ cells increased. Aged or young blood CXCR5 + CD $4+$ Tfh cells constitutively expressed CD28, and were resting $\mathrm{T}$ memory cells. There were similar frequencies of Th characterized cytokine production such as IL-21, IFN- $\gamma$, IL-4, IL-17 or IL-22 by aged and young Tfh cells. However, aged PBMCs produced significantly higher amount of IL-21. Future studies should observe whether there are defects of aged Tfh cells in helping B cells to produce Abs.

\section{Materials and methods}

\section{Ethics statement}

This study was conducted in compliance with the Declaration of Helsinki and applicable national laws and regulations, and was approved by the ethics committee of Guangzhou Medical University (Guangzhou, China) and the ethics committee of Guangdong General Hospital, Guangdong Academy of Medical Sciences (Guangzhou, China). And written informed consent was obtained from all healthy aged and young donors or the parents of young children who participated in this study. 


\section{Subjects}

Healthy young (age range: 22-39 years old, average age: 29.5 , male: $n=25$, female: $n=20$ ) and elderly (age range: $60-86$ years old, averaged age: 71.0, male: $n=19$, female: $n=24$,) volunteers were critically selected on the basis of clinical records and laboratory examinations from Guangdong General Hospital. Healthy young children (age: 0.8-2.8 years old, average age: 2.1 years old, 4 girls, 4 boys) were recruited from Guangdong General Hospital. We regarded subjects as being healthy if they had no neoplastic or autoimmune diseases, EBV, HBV, HCV, TB infections, or serious prior illnesses, were receiving on no medication other than anti-hypertensive medication. Routine laboratory examinations of the serum of these individuals were performed to examine the liver and kidney functions. We did not investigate whether the subjects had previous infection with cytomegalovirus (CMV) (Prevalence of CMV infection is $90-100 \%$ in developing countries) [49].

\section{Reagents}

Anti-CD4 PerCP, anti-CD4 PerCP-cy5.5, anti-CD3 APC, anti-CXCR5-Alexafluor 488, anti-CD45RO PE, anti-CCR7 PE, anti-CD28 PE, anti-CD69 PE, antiHLA-DR PE, anti-IL-21 PE, anti-IL-4 PE, anti-IFN- $\gamma$ APC and isotype-matched control mAbs were purchased from BD PharMingen (San Diego, CA, USA). Anti-IL-17 APC and ELISA for determining IL-21 was purchased from eBioscience (San Diego, CA, USA). Anti-IL-22 PE was purchased from R \& D Systems (Abingdon, UK). PMA, ionomycin (INO), saponin and Brefeldin A were purchased from Sigma-Aldrich (Fluka, Sigma, USA).

\section{Cell surface staining}

Heparinized blood was collected, then 50 ul blood per tube was added, and anti-CD4 PerCP, anti-CD3 APC, anti-CXCR5-Alexafluor488, anti-CD45RO PE, antiCCR7 PE, anti-CD28 PE, anti-CD69 PE, anti-HLA-DR $\mathrm{PE}$, and isotype-matched control mAbs were added into tube and mixed, incubated 15-20 minutes at room temperature. Erythrocytes were lysed with ammonium chloride $\left(\mathrm{NH}_{4} \mathrm{Cl}\right)$ for 5-8 minutes. Cells were washed, re-suspended with staining buffer, and detected by FACS Calibur (BD Biosciences, San Jose, CA). The data were analyzed using FlowJo software (Tree Star, Ashland, OR, USA). Leukocyte count and differential were determined with a routine hematology analyzer (COULTER LH780, Beckman Coulter Inc., USA). The absolute counts of each lymphocyte subpopulation were calculated by multiplying the relative size of the lymphocyte subpopulation and the absolute lymphocyte count.

\section{PBMCs preparation}

Peripheral blood mononuclear cells (PBMCs) were isolated from heparinized blood of healthy donors using FicollHypaque density gradient centrifugation, and washed twice in Hank's balanced salt solution. These cells were finally adjusted to a final concentration of $2 \times 10^{6} / \mathrm{ml}$ in complete RPMI 1640 medium (GIBCO, Grand Island, NY, USA) supplemented with 10\% FCS (Sijiqing, China), $100 \mathrm{U} / \mathrm{mL}$ penicillin, $100 \mathrm{mg} / \mathrm{mL}$ streptomycin, $50 \mathrm{mM}$ 2-mercaptoethanol, and $2 \mathrm{mM}$ L-glutamine (all from GIBCO).

\section{Intracellular cytokine staining}

PBMCs were stimulated with PMA (20 ng/ml) and ionomycin $(1 \mathrm{ug} / \mathrm{ml})$ for $4-6$ hours at $37^{\circ} \mathrm{C}$ in a $5 \% \mathrm{CO}_{2}$ humidified atmosphere. At the end of first hour during the incubation, brefeldin A (BFA, $10 \mu \mathrm{g} / \mathrm{ml}$ ) was added into the culture. The cells were collected, washed twice with cold PBS, and cells surface staining ofanti-CD4 PerCPcy5.5, anti-CXCR5-Alexafluor 488 was added for 20$30 \mathrm{~min}$. Cells were washed with PBS, fixed with $4 \%$ paraformaldehyde and re-suspended in permeabilization buffer (PBS containing $0.1 \%$ saponin and 0.5\% BSA). After incubation at $4{ }^{\circ} \mathrm{C}$ for 2 hours or overnight, intracellular cytokine staining of Anti-IL-21 PE, Anti-IL-22 PE, Anti-IL-4 PE, Anti-IL-17 APC, anti-IFN- $\gamma$ APC, and isotype-matched control $\mathrm{mAbs}$ were added and incubated at $4^{\circ} \mathrm{C}$ for $25-30 \mathrm{~min}$. Cells were washed with PBS, resuspended in cold staining buffer, and detected by FACSCalibur (BD Biosciences, San Jose, CA). The data were analyzed using FlowJo software (Tree Star, Ashland, OR, USA).

\section{Cell culture and ELISA for IL-21}

PBMCs were prepared, adjusted to a final concentration of $2 \times 10^{6} / \mathrm{ml}$, stimulated with PMA and inomycin or medium, and cultured for 3 days. Cell free culture supernatants were harvested and IL-21 was quantified by enzyme-linked immunosorbent assay (ELISA) according to the manufacturer's instruction. The sensitive of ELISA kit was $31 \mathrm{pg} / \mathrm{ml}$ for IL-21.

\section{Statistical analysis}

Comparison between two groups was assessed by Mann Whitney test for two-tails (except Figure 7, performed by paired $t$ test for two tails). P value of $<0.05$ was considered to be statistically significant. Data were expressed with mean with SEM in results. All statistical analyses were performed using GraphPad Prism (version 5.0 Software Inc, San Diego, CA, USA).

\section{Competing interests}

The authors declare that they have no competing interests. 


\section{Authors' contributions}

Conceived and designed the experiments: ES MZ. Performed the experiments: MZ RZ HG ZL FL. Analyzed the data: MZ TL YL. Contributed reagents/materials/analysis tools: ES MZ XC FH. Wrote the paper: ES RZ. All authors read and approved the final manuscript.

\section{Acknowledgements}

This work was supported by The Natural Science Foundation of Guangdong province (No. S2011040003741, S2012040006672), The Science and Technology Planned Project of Bureau of Education of Guangzhou (No. 10A159), Distinguished Young Talents in Higher Education of Guangdong province (No. LYM10111), the Guangzhou city-level key disciplines and specialties of Immunology (No. B127007), and Doctoral Fund of Ministry of Education of China (No. 20124423120003), Science and Technology Planning Project of Guangdong Province (2013). The funders had no role in study design, data collection and analysis, decision to publish, or preparation of the manuscript.

\section{Author details}

'Department of Pathology and Laboratory Medicine, Guangdong General Hospital, Guangdong Academy of Medical Sciences, Guangzhou 510080, China. ${ }^{2}$ Department of Pathogenic Biology and Immunology, Guangzhou Hoffmann Institute of Immunology, School of Basic Sciences, Guangzhou Medical University, Guangzhou 510182, China. ${ }^{3}$ Department of Obstetrics and Gynecology, The Third Affiliated Hospital of Guangzhou Medical University, Guangzhou 510150, China.

Received: 14 May 2014 Accepted: 18 August 2014

Published: 23 August 2014

\section{References}

1. McElhaney JE, Effros RB: Immunosenescence: what does it mean to health outcomes in older adults? Curr Opin Immunol 2009, 21:418-424.

2. Weinberger B, Schirmer M, MatteucciGothe R, Siebert U, Fuchs D, GrubeckLoebenstein B: Recall responses to tetanus and diphtheria vaccination are frequently insufficient in elderly persons. PLoS One 2013, 8:e82967.

3. Pawelec G, Solana R: Immunosenescence. Immunol Today 1997, 10:514-516.

4. Shaw AC, Goldstein DR, Montgomery RR: Age-dependent dysregulation of innate immunity. Nat Rev Immunol 2013, 13:875-887.

5. Camous X, Pera A, Solana R, Larbi A: NK cells in healthy aging and age-associated diseases. J Biomed Biotechnol 2012, 2012:195956.

6. Gayoso I, Sanchez-Correa B, Campos C, Alonso C, Pera A, Casado JG, Morgado S, Tarazona R, Solana R: Immunosenescence of human natural killer cells. J Innate Immun 2011, 3:337-343.

7. Agrawal A, Tay J, Ton S, Agrawal S, Gupta S: Increased reactivity of dendritic cells from aged subjects to self-antigen, the human DNA. J Immunol 2009, 182:1138-1145.

8. Panda A, Qian F, Mohanty S, van Duin D, Newman FK, Zhang L, Chen S, Towle V, Belshe RB, Fikrig E, Allore HG, Montgomery RR, Shaw AC: Age-associated decrease in TLR function in primary human dendritic cells predicts influenza vaccine response. J Immunol 2010, 184:2518-2527.

9. Wong C, Goldstein DR: Impact of aging on antigen presentation cell function of dendritic cells. Curr Opin Immunol 2013, 25:535-541.

10. Fülöp T, Larbi A, Pawelec G: Human T cell aging and the impact of persistent viral infections. Front Immunol 2013, 4:271.

11. Haynes L, Maue AC: Effects of aging on T cell function. Curr Opin Immunol 2009, 21:414-417.

12. Krone $\mathrm{CL}$, van de Groep K, Trzciński K, Sanders EA, Bogaert D: Immunosenescence and pneumococcal disease: an imbalance in hostpathogen interactions. Lancet Respir Med 2014, 2:141-153.

13. Eilers R, Krabbe PF, van Essen TG, Suijkerbuijk A, van Lier A, de Melker HE: Assessment of vaccine candidates for persons aged 50 and older: a review. BMC Geriatr 2013, 13:32. doi: 10.1186/1471-2318-13-32.

14. Goodwin K, Viboud C, Simonsen L: Antibody response to influenza vaccination in the elderly: a quantitative review. Vaccine 2006, 24:1159-1169.

15. Kogut I, Scholz JL, Cancro MP, Cambier JC: B cell maintenance and function in aging. Semin Immunol 2012, 24:342-349.

16. Ademokun A, Wu YC, Dunn-Walters D: The ageing B cell population: composition and function. Biogerontology 2010, 11:125-137.

17. Frasca D, Blomberg BB: Aging affects human B cell responses. J Clin Immunol 2011, 31:430-435
18. Buffa S, Bulati M, Pellicanò M, Dunn-Walters DK, Wu YC, Candore G, Vitello $S$, Caruso C, Colonna-Romano G: B cell immunosenescence: different features of naive and memory B cells in elderly. Biogerontology 2011, 12:473-483.

19. Frasca D, Diaz A, Romero M, Landin AM, Phillips M, Lechner SC, Ryan JG, Blomberg BB: Intrinsic defects in B cell response to seasonal influenza vaccination in elderly humans. Vaccine 2010, 28:8077-8084.

20. Eaton SM, Burns EM, Kusser K, Randall TD, Haynes L: Age-related defects in CD4 T cell cognate helper function lead to reductions in humoral responses. J Exp Med 2004, 200:1613-1622.

21. Haynes L, Eaton SM: The effect of age on the cognate function of CD4+ T cells. Immunol Rev 2005, 205:220-228.

22. Lefebvre JS, Maue AC, Eaton SM, Lanthier PA, Tighe M, Haynes L: The aged microenvironment contributes to the age-related functional defects of CD4 T cells in mice. Aging Cell 2012, 11:732-740.

23. Tangye SG, Ma CS, Brink R, Deenick EK: The good, the bad and the ugly - TFH cells in human health and disease. Nat Rev Immunol 2013, 13:412-426.

24. Crotty S: Follicular helper CD4 T cells (TFH). Annu Rev Immunol 2011, 29:621-663.

25. Nurieva RI, Chung Y, Martinez GJ, Yang XO, Tanaka S, Matskevitch TD, Wang $\mathrm{YH}$, Dong C: Bcl6 mediates the development of T follicular helper cells. Science 2009, 325:1001-1005.

26. Johnston RJ, Poholek AC, DiToro D, Yusuf I, Eto D, Barnett B, Dent AL, Craft J, Crotty S: Bcl6 and Blimp-1 are reciprocal and antagonistic regulators of T follicular helper cell differentiation. Science 2009, 325:1006-10010.

27. Nurieva RI, Chung Y, Hwang D, Yang XO, Kang HS, Ma L, Wang YH, Watowich SS, Jetten AM, Tian Q, Dong C: Generation of T follicular helper cells is mediated by interleukin-21 but independent of T helper 1, 2, or 17 cell lineages. Immunity 2008, 29:138-149.

28. Morita R, Schmitt N, Bentebibel SE, Ranganathan R, Bourdery L, Zurawski G, Foucat E، Dullaers M, Oh S, Sabzghabaei N, Lavecchio EM, Punaro M, Pascual V, Banchereau J, Ueno H: Human blood CXCR5 (+) CD4 (+) T cells are counterparts of $\mathrm{T}$ follicular cells and contain specific subsets that differentially support antibody secretion. Immunity 2011, 34:108-121.

29. McDonald KG, Leach MR, Huang C, Wang C, Newberry RD: Aging impacts isolated lymphoid follicle development and function. Immun Ageing 2011, 8:1.

30. Hirokawa K, Utsuyama M, Hayashi Y, Kitagawa M, Makinodan T, Fulop T: Slower immune system aging in women versus men in the Japanese population. Immun Ageing 2013, 10:19.

31. Provinciali M, Moresi R, Donnini A, Lisa RM: Reference values for CD4+ and CD8+ T lymphocytes with naïve or memory phenotype and their association with mortality in the elderly. Gerontology 2009, 55(3):314-321.

32. Chng WJ, Tan GB, Kuperan P: Establishment of adult peripheral blood lymphocyte subset reference range for an Asian population by singleplatform flow cytometry: influence of age, sex, and race and comparison with other published studies. Clin Diagn Lab Immunol 2004, 11:168-173.

33. Wertheimer AM, Bennett MS, Park B, Uhrlaub JL, Martinez C, Pulko V, Currier NL, Nikolich-Žugich D, Kaye J, Nikolich-Žugich J: Aging and cytomegalovirus infection differentially and jointly affect distinct circulating T cell subsets in humans. J Immunol 2014, 192:2143-2155.

34. Sallusto F, Geginat J, Lanzavecchia A: Central memory and effector memory T cell subsets: function, generation, and maintenance. Annu Rev Immunol 2004, 22:745-763.

35. Schaerli P, Willimann K, Lang AB, Lipp M, Loetscher P, Moser B: CXC chemokine receptor 5 expression defines follicular homing $T$ cells with $B$ cell helper function. J Exp Med 2000, 192:1553-1562.

36. Breitfeld D, Ohl L, Kremmer E, Ellwart J, Sallusto F, Lipp M, Förster R: Follicular B helper T cells express CXC chemokine receptor 5, localize to $B$ cell follicles, and support immunoglobulin production. J Exp Med 2000 192:1545-1552.

37. Kim CH, Rott LS, Clark-Lewis I, Campbell DJ, Wu L, Butcher EC: Subspecialization of CXCR5+ T cells: B helper activity is focused in a germinal center-localized subset of CXCR5+ T cells. J Exp Med 2001, 193:1373-1381.

38. Farber DL, Yudanin NA, Restifo NP: Human memory T cells: generation, compartmentalization and homeostasis. Nat Rev Immunol 2014 $14: 24-35$.

39. Linterman MA, Vinuesa CG: Signals that influence $T$ follicular helper cell differentiation and function. Semin Immuno Pathol 2010, 32:183-196.

40. Weng NP, Akbar AN, Goronzy J: CD28 (-) T cells: their role in the age-associated decline of immune function. Trends Immunol 2009, 30:306-312. 
41. Yi JS, Cox MA, Zajac AJ: Interleukin-21: a multifunctional regulator of immunity to infections. Microbes Infect 2010, 12:1111-1119.

42. Liu SM, King C: IL-21-producing Th cells in immunity and autoimmunity. J Immunol 2013, 191:3501-3506.

43. Agrawal A, Su H, Chen J, Osann K, Agrawal S, Gupta S: Increased IL-21 secretion by aged CD4 + T cells is associated with prolonged STAT-4 activation and CMV seropositivity. Aging (Albany NY) 2012, 4:648-659.

44. Onoda T, Rahman M, Nara H, Araki A, Makabe K, Tsumoto K, Kumagai I, Kudo T, Ishii N, Tanaka N, Sugamura K, Hayasaka K, Asao H: Human CD4+ central and effector memory T cells produce IL-21: effect on cytokine-driven proliferation of CD4+ T cell subsets. Int Immunol 2007, 19:1191-1199.

45. Yu M, Li G, Lee WW, Yuan M, Cui D, Weyand CM, Goronzy JJ: Signal inhibition by the dual-specific phosphatase 4 impairs T cell-dependent B-cell responses with age. Proc Natl Acad Sci U S A 2012, 109:E879-E888.

46. Sarra M, Franzè E, Pallone F, Monteleone G: Targeting interleukin-21 in inflammatory diseases. Expert Opin Ther Targets 2011, 15:695-702.

47. Lee N, Shin MS, Kang I: T-cell biology in aging, with a focus on lung disease. J Gerontol A Biol Sci Med Sci 2012, 67:254-263.

48. Lee JS, Lee WW, Kim SH, Kang Y, Lee N, Shin MS, Kang SW, Kang I: Age-associated alteration in naive and memory Th17 cell response in humans. Clin Immunol 2011, 140:84-91.

49. Fang FQ, Fan QS, Yang ZJ, Peng YB, Zhang L, Mao KZ, Zhang Y, Ji YH: Incidence of cytomegalovirus infection in Shanghai, China. Clin Vaccine Immunol 2009, 16:1700-1703.

doi:10.1186/1742-4933-11-12

Cite this article as: Zhou et al:: The effect of aging on the frequency, phenotype and cytokine production of human blood CD4 + CXCR5 + T follicular helper cells: comparison of aged and young subjects. Immunity \& Ageing 2014 11:12

\section{Submit your next manuscript to BioMed Central and take full advantage of:}

- Convenient online submission

- Thorough peer review

- No space constraints or color figure charges

- Immediate publication on acceptance

- Inclusion in PubMed, CAS, Scopus and Google Scholar

- Research which is freely available for redistribution 